\title{
Exploration the Mechanism of Doxorubicin-Induced Heart Failure in Rats by Integration of Proteomics and Metabolomics Data
}

\author{
Yu Yuan ${ }^{\dagger}$, Simiao $\mathrm{Fan}^{\dagger}$, Lexin Shu ${ }^{\dagger}$, Wei Huang ${ }^{\dagger}$, Lijuan Xie, Chenghao Bi, Hongxin Yu, \\ Yuming Wang * and Yubo Li*
}

Department of Traditional Chinese Medicine, Tianjin University of Traditional Chinese Medicine, Tianjin, China

\section{OPEN ACCESS}

Edited by:

Antonella De Angelis, University of Campania Luigi Vanvitelli,

Reviewed by:

Beshay Zordoky,

University of Minnesota, United States

Ada Popolo,

University of Salerno, Italy

*Correspondence:

Yuming Wang

wangyuming226@163.com

Yubo Li

yuboli1@163.com

${ }^{\dagger}$ These authors have contributed equally to this work and share first authorship

Specialty section: This article was submitted to Cardiovascular and Smooth Muscle

Pharmacology,

a section of the journal

Frontiers in Pharmacology

Received: 02 September 2020

Accepted: 02 November 2020

Published: 10 December 2020

Citation:

Yuan Y, Fan S, Shu L, Huang W, Xie L, Bi C, Yu H, Wang $Y$ and Li $Y$ (2020)

Exploration the Mechanism of Doxorubicin-Induced Heart Failure in Rats by Integration of Proteomics and Metabolomics Data.

Front. Pharmacol. 11:600561. doi: 10.3389/fphar.2020.600561
Heart failure is a common systemic disease with high morbidity and mortality worldwide. Doxorubicin (DOX) is a commonly used anthracycline broad-spectrum antitumor antibiotic with strong antitumor effect and definite curative effect. However, cardiotoxicity is the adverse reaction of drug dose cumulative toxicity, but the mechanism is still unclear. In this study, proteomics and metabonomics techniques were used to analyze the tissue and plasma of DOX-induced heart failure (HF) in rats and to clarify the molecular mechanism of the harmful effects of DOX on cardiac metabolism and function in rats from a new point of view. The results showed that a total of 278 proteins with significant changes were identified by quantitative proteomic analysis, of which 118 proteins were significantly upregulated and 160 proteins were significantly downregulated in myocardial tissue. In the metabonomic analysis, 21 biomarkers such as L-octanoylcarnitine, alpha-ketoglutarate, glutamine, creatine, and sphingosine were detected. Correlation analysis showed that DOX-induced HF mainly affected phenylalanine, tyrosine, and tryptophan biosynthesis, D-glutamine and D-glutamate metabolism, phenylalanine metabolism, biosynthesis of unsaturated fatty acids, and other metabolic pathways, suggesting abnormal amino acid metabolism, fatty acid metabolism, and glycerol phospholipid metabolism. It is worth noting that we have found the key upstream target of DOX-induced HF, PTP1B, which inhibits the expression of HIF-1a by inhibiting the phosphorylation of IRS, leading to disorders of fatty acid metabolism and glycolysis, which together with the decrease of Nrf2, SOD, Cytc, and AK4 proteins lead to oxidative stress. Therefore, we think that PTP1B may play an important role in the development of heart failure induced by doxorubicin and can be used as a potential target for the treatment of heart failure.

Keywords: doxorubicin, heart failure, proteomics, metabonomics, protein tyrosine-protein phosphatase nonreceptor type 1

\section{INTRODUCTION}

Heart disease has a high mortality and morbidity worldwide. Clinically, anthracyclines are a major reason of cardiotoxicity (Feijen et al., 2019). For example, doxorubicin (DOX) is an effective anticancer agent for the treatment of solid tumors and hematological malignancies, which can cause pathological and physiological damage to the heart, such as progressive cardiac dilation and systolic 
dysfunction, and subsequently lead to heart failure (HF) (Eisenberg et al., 2018; Russo et al., 2019; Maurer and Packer, 2020). Studies have shown that the occurrence of HF caused by DOX is related to cardiomyocyte death caused by mitochondrial swelling, myocardial fibrosis, or cardiac contraction/diastole (Zhang K. W. et al., 2018; Galán-Arriola, 2019). Rochette et al. showed in the study that DOX caused a significant reduction in left ventricular ejection fraction, leading to congestive $\mathrm{HF}$ (Rochette et al., 2015). In the treatment of breast cancer patients, HF is the main cause of death due to the use of anthracycline drugs, which can be manifested as, under the condition of no abnormal load, left ventricular dilation and poor function lead to cardiac systolic dysfunction (Zhang et al., 2020). Based on the above studies, when anthracycline is used as a chemotherapeutic agent, cardiac toxicity has become the main limiting factor, and the discovery, prevention, and treatment of it will be an important issue.

In medicine, proteomics and metabonomics are used to help identify patients at risk of disease, detect and identify various molecules at the level of proteins and metabolites, and study their functions and interrelationships among various molecules (Mato et al., 2014). The combined analysis of the two techniques can link genotype and phenotype to help determine the causal mechanism of DOX-induced HF and provide a new strategy for the discovery and effective treatment of HF (Hoffman et al., 2017).

Here, we used ultra-high performance liquid chromatographytandem quadrupole time-of-flight mass spectrometry (UPLC-QTOF/MS) to analyze DOX-induced HF in rats, to explain the toxicological pathways of DOX to the heart, especially the combined proteomic technology, to provide more information for the study of its pathogenesis. We also studied the potential upstream targets for the development of HF.

\section{MATERIALS AND METHODS}

\subsection{Chemicals}

Doxorubicin (Solarbio, Beijing, China), formaldehyde (Solarbio, Beijing, China), heparin sodium (Solarbio, Beijing, China), acetonitrile (Merck, Germany), formic acid (CNW, Germany), BCA Kit (Solarbio, Shangahi, China), Tris (Sigma, USA), SDS (Bio-Rad, USA), phenylmethylsulfonyl fluoride (Solarbio, Beijing), phosphatase inhibitor (Solarbio, Beijing, China), RIPA buffer (Solarbio, Beijing, China), PTP1B (Abcam, USA), IRS-1, P-IRS, HK2, HIF-1a (Cell Signaling Technology, Inc., USA), Nrf2 (Abcam, USA), NH4HCO3 (Sigma, USA), High pH Reversed-Phase Peptide Fractionation Kit (Pierce, USA), TMT 6/10 plex Isobaric Label Reagent (Thermo, USA) were used.

\subsection{Animals}

A total of 35 male Wistar rats (180-200 g) were purchased from Beijing Weitong Lihua Experimental Animal Technology Co., Ltd., license number: SCXK (Beijing) 2016-0006. After one week of adaptive feeding, the rats were acclimatized to a 12-hour light/ dark cycle in a controlled environment with a temperature of approximately $23 \pm 2^{\circ} \mathrm{C}$ and a relative humidity of $35 \pm 5 \%$. All animals received care and raising with standard food and tap water. The rats were randomly divided into a treatment group (Dox group) and a control group (NS group). The Dox group was injected intraperitoneally with DOX $(3 \mathrm{mg} / \mathrm{kg})$ once a week, and the NS group was injected with the same amount of normal saline intraperitoneally, for 6 weeks.

\subsection{Compliance With Ethical Standards}

This study was approved by the Institutional Animal Care and Use Committee of Tianjin University of Traditional Chinese Medicine (IACUC) and was conducted in accordance with the guidelines of the National Institutes of Health Animal Care and Use Committee.

\subsection{Biochemical and Pathological Observation}

After induction of anesthesia with 5\% chloral hydrate, the longaxis parasternal sections of rats were measured using an ultrasound Doppler instrument, and cardiac function indexes were recorded. Ejection fraction (EF) was used as a parameter to determine the establishment of HF. From each serum sample, $100 \mu \mathrm{L}$ was extracted and measured in a fully automated biochemical instrument, the data of lactate dehydrogenase $(\mathrm{LDH})$, troponin kinase $(\mathrm{CK})$, and creatine kinase isoenzymes (CK-MB) were recorded.

Small sections of heart tissues of NS group and DOX group were fixed with $10 \%$ formaldehyde, embedded in paraffin, and then prepared into $5 \mu \mathrm{m}$-thick sections. Hematoxylin and eosin (H\&E) staining was performed in heart sections to visualize the pathological manifestations under the microscope.

\subsection{Metabolomics Analysis}

We analyzed the plasma of rats in NS group and DOX group by metabonomics. Plasma samples of rats were centrifuged at $4^{\circ} \mathrm{C}$ and $3500 \mathrm{rpm}$ for $10 \mathrm{~min}$. Each sample was fed with $100 \mu \mathrm{L}$ of plasma, added to $300 \mu \mathrm{L}$ acetonitrile $(1: 3 \mathrm{v} / \mathrm{v})$, and vortexed for $1 \mathrm{~min}$, and then put in ultrasonic ice water bath for $10 \mathrm{~min}$ and in the freezing centrifuge (ALLLEGRATM-64R; American) in $13,000 \mathrm{rmp}, 4^{\circ} \mathrm{C} / 15 \mathrm{~min}$, retaining supernatant. QC samples were prepared to contain biological information of all samples.

To further assess the alterations of metabolites in rats treated with DOX, endogenous metabolites were analyzed by Waters Xevo G2-S UPLC-Q-TOF/MS (Waters, USA). $10 \mu \mathrm{L}$ of sample solution was injected onto an ACQUITY UPLC BEH C18 $(2.1 \mathrm{~mm} \times 100 \mathrm{~mm}, 1.7 \mu \mathrm{m}$, Waters $)$ at $45^{\circ} \mathrm{C}$ and the flow rate was $0.3 \mathrm{ml} / \mathrm{min}$. The optimal mobile phase consisted of a linear gradient system of a) $0.1 \%$ formic acid in water and b) $0.1 \%$ formic acid in acetonitrile, $0-8.5 \mathrm{~min}, 1-25 \% \mathrm{~B} ; 8.5-11.0 \mathrm{~min}$, 25-50\% B; 11.0-13.0 min, 50-1\% B; 13.0-15.0 min, 1-1\% B. In addition, the QC samples contained most information of whole plasma samples.

The mass spectrum optimal conditions of analysis were as follows: Electrospray ionization source was used, in positive and negative ionization modes, the capillary voltage was $3.0 \mathrm{kV}$, drying gas temperature was set at $325^{\circ} \mathrm{C}$, drying gas flow was $10 \mathrm{ml} / \mathrm{min}$, desolvation gas flow was $600 \mathrm{~L} / \mathrm{h}$, source temperature 
was $120^{\circ} \mathrm{C}$, desolvation temperature was $350^{\circ} \mathrm{C}$, and cone gas flow was $50 \mathrm{~L} / \mathrm{h}$. The quadrupole scan range was set at $\mathrm{m} / \mathrm{z}$ 50-1000 Da.

\subsection{Quantitative Proteomics Analysis}

Rat myocardial tissue samples were frozen with liquid nitrogen, ground, and crushed with SDT lysate (4\% SDS, $100 \mathrm{mM}$ Tris$\mathrm{HCl}, 1 \mathrm{mM}$ DTT, $\mathrm{pH}$ 7.6) in MP homogenizer. Protein quantification was performed by BCA method. Each sample was $30 \mathrm{ml}$ protein solution, enzymatically hydrolyzed by filter aided proteome preparation (FASP) method (Marwick, 2015), and the peptide segment was quantified under OD280. Each sample was labeled according to the instructions of Thermo TMT labeling kit. The labeled samples were combined and subjected to fractionation (See Supplementary $\mathbf{1}$ for detailed treatment).

Then each fraction was injected for HPLC analysis, followed by LC-MS/MS analysis performed on a Q Exactive mass spectrometer (Thermo Scientific) that was coupled to Easy nLC (Thermo Fisher Scientific). Buffer A is $0.1 \%$ formic acid buffer; $\mathrm{B}$ is $84 \%$ acetonitrile and $0.1 \%$ formic acid aqueous solution. The chromatographic columns used were packed with nanoViper C18 (Thermo Scientific Acclaim PepMap100, $100 \mu \mathrm{m} \times 2 \mathrm{~cm}, 3 \mu \mathrm{m}, 100 \mathrm{~A}$ ) balanced by $95 \%$ A solution at a flow rate of $300 \mathrm{~nL} / \mathrm{min}$. The peptides were eluted using a linear gradient $\quad(0-80 \mathrm{~min}, \quad 0-55 \% \quad \mathrm{~B} ; \quad 80-85 \mathrm{~min}, \quad 55-100 \% \quad \mathrm{~B}$; 85-90 min, 100\% B).

The detection mode is positive ion, the scanning range of parent ion is $300-1800 \mathrm{~m} / \mathrm{z}$, the resolution of primary mass spectrometry is 70,000 at $200 \mathrm{~m} / \mathrm{z}$, the target of Automatic Gain Control is 1e6, Maximum IT is $50 \mathrm{~m} \mathrm{~s}$, and the Dynamic Exclusion time is $60.0 \mathrm{~s}$. The mass-charge ratio of peptides and peptide fragments is collected according to the following methods: 20 fragment spectra (MS2 scan) were collected after each full scan, MS2 Activation Type was HCD, isolation window was $2 \mathrm{~m} / \mathrm{z}$, and secondary mass spectrometry resolution was 17,500 at $200 \mathrm{~m} / \mathrm{z}$ (TMT 6-plex) or 35,000 at $200 \mathrm{~m} / \mathrm{z}$ (TMT 10-plex), $30 \mathrm{eV}$ for Normalized Collision Energy and $0.1 \%$ for Underfill.

\subsection{Data Processing}

MassLynx software (Waters, Version 4.1) was used to extract peak values and correct the original mass spectra data of metabonomics for principal component analysis (PCA) and partial least squares-discriminant analysis (PLS-DA). The standards were variable importance in the projection (VIP) > 1.5 and T-test $(p<0.05)$ to select potential biomarkers. Subsequently according to the HMDB database (http://www. hmdb.ca), the differential metabolites were further identified by $\mathrm{m} / \mathrm{z}$ values and Mass Spectrometry Fragmentation. The identified metabolites were analyzed by receiver operating characteristic curve and clustering analysis software was used according to the relative content of each biomarker (http://www. metaboanalyst.ca/) to generate a heat map to evaluate the diagnostic ability of metabolic markers. Pathway and visualization analysis was done using the KEGG pathway database (http://mirror.MetaboAnalyst.ca/).
For the tandem mass tag (TMT) proteomic data, MS/MS spectra were searched using MASCOT engine (Matrix Science, London, UK, version 2.2) embedded into Proteome Discoverer 1.4 (Supplementary 1). LC/MS raw data were searched in the UniProt-reviewed rat protein database and differentially expressed proteins were screened by the criteria of upregulation greater than 1.2 times or downregulation less than 0.83 and $p$ value less than 0.05 . All identified proteins were annotated with Blast2GO for GO function (Database version: go_201504.obo, www.geneontology.org), and then differentially expressed proteins were analyzed by Fisher's exact test for GO functional enrichment and bioinformatics. Next, through the KEGG (http://www.kegg.jp/) pathway enrichment analysis of significantly differentially expressed proteins, it can help us to understand the metabolic or signal pathways that these proteins may be involved in.

\subsection{Western Blot Analysis}

The $50 \mathrm{mg}$ heart tissue was added to the phenylmethylsulfonyl fluoride (PMSF), phosphatase inhibitor, and RIPA buffer and lysed and cracked for $30 \mathrm{~min}$ on the ice to ensure complete cleavage. The protein was quantified with the BCA protein assay kit and then protein was separated by $8 \%$ SDS-PAGE. The proteins were transferred to polyvinylidene difluoride (PVDF) membrane and incubated overnight with primary antibodies against PTP1B, IRS-1, P-IRS, HK2, HIF-1 a, and Nrf2, followed by incubation with $\beta$-actin and NADPH, which served as an internal control.

\subsection{Statistical Analysis}

The analysis of related data was performed using SPSS software (version 17.0). The experimental data are expressed as the mean \pm SD. Data comparison between various experimental groups was performed by T-test or one-way ANOVA. ${ }^{*} p<0.05$, ${ }^{\star *} p<0.01$.

\section{RESULTS}

\subsection{Effect of Doxorubicin on Heart Biochemical Parameters and Pathological Situations of Rat}

DOX is an effective cancer chemotherapy agent, which can cause pathological and physiological HF (Fu et al., 2016). Echocardiography directly reflected the ejection capacity, systolic and diastolic function of the heart, and played an irreplaceable role in the diagnosis of cardiotoxicity. We compared the echocardiograms. A significant elevation of serum diastolic left ventricular volume (LVID, d) and systolic left ventricular volume (LVID, s) was observed in DOX group compared with NS group over the entire time course, whereas the levels of EF, left ventricular diameter shortening rate (FS), left ventricular posterior wall systolic thickness (LVPW, s), and left ventricular posterior wall diastolic thickness (LVPW, d) were decreased, as shown in Figure 1A. In addition, we also compared the changes of serum CK, CK-MB, and LDH between the DOX group and the NS group (Figure 1B) and evaluated the degree of 

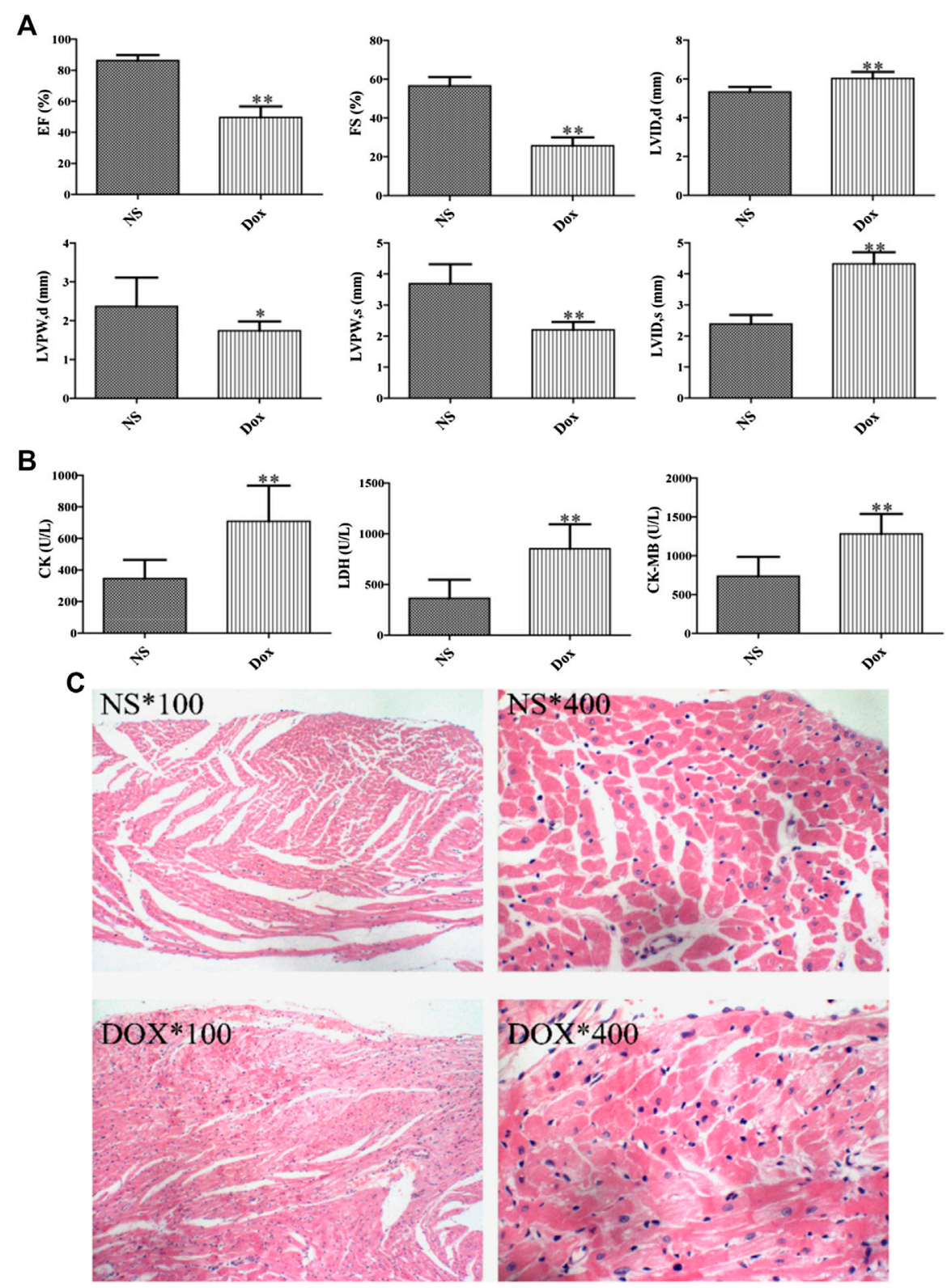

FIGURE 1 | Effects of DOX-induced heart pathology and biochemistry changes in heart failure. (A) Echocardiographic evaluation of rat hearts. (B) Biochemical indices in rat serum. (C) Staining results of rat cardiomyocytes. $\left({ }^{\star} p<0.05,{ }^{\star \star} p<0.01 .{ }^{\star}, 100\right.$ : magnification $\times 100 ;{ }^{*}, 400$ : magnification $\left.\times 400\right)$.

cardiotoxicity of DOX in rats. In conclusion, it indicated that the rats presented the typical pathological features of myocardial damage.

The damage severity was further verified by H\&E staining of myocardial cell. In Figure 1C, the rat myocardial cells in the NS group are arranged orderly and the texture structure is clear. On the contrary, the myocardial cells in the DOX group had more significant edema, some myocardial nuclei were large and stained deeply, and there were many vacuoles near the nuclei, the obvious infiltration of inflammatory cells can be seen in the stroma, and the myocardial fibers are dissolved and broken. Consequently, combined with the histopathological manifestation results, DOX can affect the normal physiological state of the heart.

\subsection{Metabolomics Analysis}

Plasma samples of rats were analyzed by UPLC-Q-TOF/MS to obtain significantly changed metabolites. The original mass spectra data were analyzed with SIMCA software (version 12.0, Sweden) for multivariate statistical analysis respectively. Then PCA (Figures 2A,E) and PLS-DA were calculated (Figures 2B,F). The results showed complete separation of the heart between the NS group and the DOX injury groups in both negative and positive ionization mode, which were described 

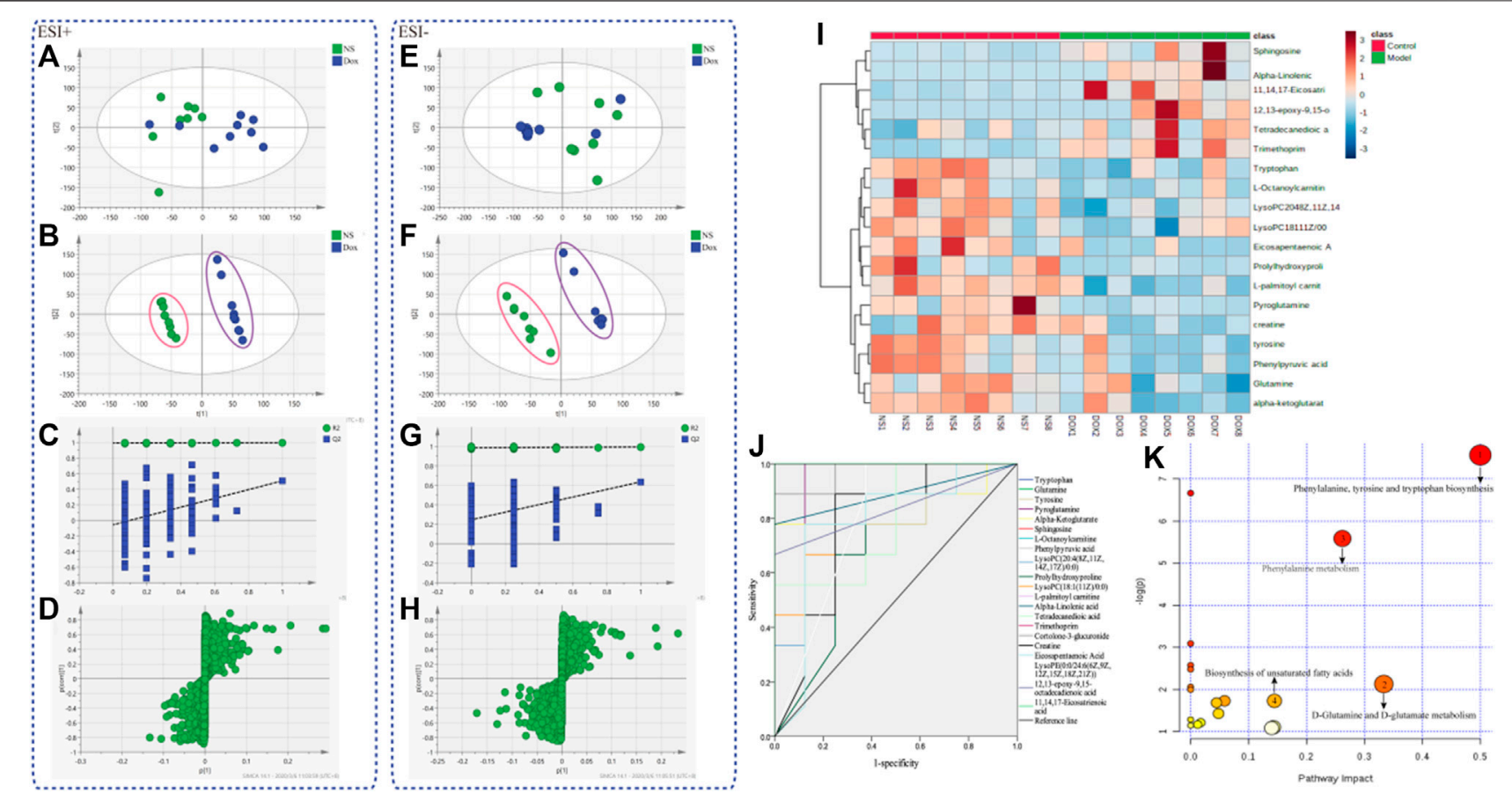

FIGURE 2 | The metabolic profiles and metabolite analysis of plasma samples. (A) PCA score plots (ESI+) for comprehensive metabonomic data of plasma samples. Models include the control and DOX-treated rat (3 mg/kg). (B) PLS-DA models (ESI+) of UPLC-Q-TOF/MS metabonomics data for NS group and models include the control and DOX-treated rat (3 mg/kg). (C) Model validation diagram in positive ion mode. (D) S-plot load diagram in positive ion mode. (E) PCA score plots (ESI-) for comprehensive metabonomic data of plasma samples. Models include the control and DOX-treated rat (3 mg/kg). (F) PLS-DA models (ESI-) of UPLC-QTOF/MS metabonomics data for NS group and models include the control and DOX-treated rat (3 mg/kg). (G) Model validation diagram in negative ion mode. (H) S-plot load diagram in negative ion mode. (I) Heat map for metabolites. (J) Diagnostic significance of ROC curve analysis for metabolites. (K) Metabolic pathway map analysis.

by high values of R2Y (0.996 and 0.996) and Q2 (0.665 and 0.665) parameters. We also performed permutation tests (Figures

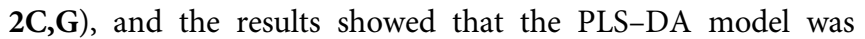
not overfitted and had high reliability. The model validation diagram is used to diagnose, and the number of tests is set to 200 . The obtained model validation diagram proves that the PLS-DA model has no overfitting and high reliability. Meanwhile, S-plot load plot (Figures 2D,H) showed that the farther the metabolites from the central origin on the " $\mathrm{S}$ " curve, the greater the VIP value and the greater the contribution. All the differential endogenous metabolites meet the requirements of VIP $>1.5$ and $p<0.05$. By further MS2 identification, 21 significantly changed ions were matched with endogenous metabolites from HMDB database (Table 1); 12 of the identified metabolites were downregulated and nine were upregulated. Next, we will diagnose the 21 differential metabolites' diagnostic significance by two ways: ROC curve and cluster analysis. The ROC curve (Figure 2J) shows that the area of the 21 metabolites under the curve is all greater than 0.7; the heat map (Figure 2I) results showed that the content changes of 21 biomarkers in the NS group and the DOX group were significantly different, indicating that they had better accuracy in the diagnosis of heart failure induced by DOX. Based on the KEGG pathway enrichment analysis and topological analysis of MetPA database (Figure $\mathbf{2 K}$ ), we can intuitively see that these differential metabolites participate in a variety of metabolic pathways, among which the metabolic pathways more influential in heart failure include the phenylalanine, tyrosine, and tryptophan biosynthesis, the D-glutamine and D-glutamate metabolism, the phenylalanine metabolism, and the biosynthesis of unsaturated fatty acids. Through these results, we found that HF mainly involves energy metabolism, amino acid metabolism, fatty acid metabolism, and glycerophospholipid metabolism disorders.

\subsection{Proteomic Analysis}

By utilization of TMT-based quantitative proteomics, we identified 29,483 peptides in the rats heart tissue samples of the two groups and identified 3,727 proteins (Supplementary 2), among which 278 proteins were significantly altered (fold change $>1.2$ or $<0.83, p<0.05)$ as a result of doxorubicin treatments. In detail, 118 proteins were upregulated and 160 proteins were downregulated in adriamycin treated group (Supplementary 2). In order to better understand which functions or biological pathways are significantly affected by biological processing, we annotated 278 differential proteins by Blast2Go software (https:// www.blast2go.com/) for GO function. It can be seen that significant changes have taken place in the top $20 \mathrm{GO}$ terms (Figure 3A): Biological processes such as the generation of precursor metabolites and ATP metabolic process; molecular functions such as glutathione binding and antioxidant activity; cellular components such as mitochondrial envelope, extracellular space, and extracellular region. Notably, to 
TABLE 1 | Potential biomarkers were selected in plasma of COX-treated rats and normal rats.

\begin{tabular}{|c|c|c|c|c|c|c|c|c|}
\hline No & $\begin{array}{c}\mathbf{T}_{R} \\
(\min )\end{array}$ & Compound & $\begin{array}{l}\text { Molecular } \\
\text { formula }\end{array}$ & $\begin{array}{l}m / z \\
\text { obsd }\end{array}$ & $\begin{array}{c}m / z \\
\text { calcd }\end{array}$ & $\begin{array}{l}\text { Error } \\
\text { (ppm) }\end{array}$ & Ion mode & $\begin{array}{c}\text { Treatment } \\
\text { group } \\
\text { vs. } \\
\text { control } \\
\text { group }\end{array}$ \\
\hline 1 & 0.93 & Tryptophan & $\mathrm{C}_{11} \mathrm{H}_{12} \mathrm{~N}_{2} \mathrm{O}_{2}$ & 227.0796 & 227.0796 & 0 & + & $\downarrow^{\star \star}$ \\
\hline 2 & 0.92 & Tyrosine & $\mathrm{C}_{9} \mathrm{H}_{11} \mathrm{NO}_{3}$ & 182.0817 & 182.0819 & 1.10 & + & $\downarrow^{*}$ \\
\hline 3 & 0.80 & Glutamine & $\mathrm{C}_{5} \mathrm{H}_{10} \mathrm{~N}_{2} \mathrm{O}_{3}$ & 169.0589 & 169.0587 & -1.18 & + & $\downarrow^{*}$ \\
\hline 4 & 0.83 & Pyroglutamine & $\mathrm{C}_{5} \mathrm{H}_{8} \mathrm{~N}_{2} \mathrm{O}_{2}$ & 129.0664 & 129.0665 & 0.77 & + & $\downarrow^{*}$ \\
\hline 5 & 11.31 & Alpha-ketoglutarate & $\mathrm{C}_{5} \mathrm{H}_{6} \mathrm{O}_{5}$ & 184.9852 & 184.9864 & 6.49 & + & $\downarrow^{\star \star}$ \\
\hline 6 & 4.41 & Sphingosine & $\mathrm{C}_{18} \mathrm{H}_{37} \mathrm{NO}_{2}$ & 300.2903 & 300.2902 & -0.33 & + & $\uparrow^{\star}$ \\
\hline 7 & 2.80 & L-Octanoylcarnitine & $\mathrm{C}_{15} \mathrm{H}_{29} \mathrm{NO}_{4}$ & 288.2175 & 288.2169 & -2.08 & + & $\downarrow^{*}$ \\
\hline 8 & 0.93 & Phenylpyruvic acid & $\mathrm{C}_{9} \mathrm{H}_{8} \mathrm{O}_{3}$ & 165.0552 & 165.0547 & -3.03 & + & $\uparrow^{*}$ \\
\hline 9 & 2.45 & Prolylhydroxyproline & $\mathrm{C}_{10} \mathrm{H}_{16} \mathrm{~N}_{2} \mathrm{O}_{4}$ & 251.1008 & 251.1014 & 2.39 & + & $\downarrow^{*}$ \\
\hline 10 & 5.37 & LysoPC(20:4 (8Z,11Z,14Z,17Z)/0:0) & $\mathrm{C}_{28} \mathrm{H}_{50} \mathrm{NO}_{7} \mathrm{P}$ & 566.3223 & 566.3218 & -0.80 & + & $\downarrow^{\star \star}$ \\
\hline 11 & 6.25 & LysoPC(18:1 (11Z)/0:0) & $\mathrm{C}_{26} \mathrm{H}_{52} \mathrm{NO}_{7} \mathrm{P}$ & 544.3379 & 544.3381 & 0.37 & + & $\uparrow^{\star}$ \\
\hline 12 & 5.48 & L-Palmitoyl carnitine & $\mathrm{C}_{23} \mathrm{H}_{45} \mathrm{NO}_{4}$ & 400.3427 & 400.3432 & 1.25 & + & $\downarrow^{\star \star}$ \\
\hline 13 & 5.66 & Alpha-linolenic acid & $\mathrm{C}_{18} \mathrm{H}_{30} \mathrm{O}_{2}$ & 279.2324 & 279.2314 & -3.58 & + & $\uparrow^{\star}$ \\
\hline 14 & 6.03 & Tetradecanedioic acid & $\mathrm{C}_{14} \mathrm{H}_{26} \mathrm{O}_{4}$ & 297.1468 & 297.15 & 10.77 & + & $\uparrow^{*}$ \\
\hline 15 & 6.28 & Trimethoprim & $\mathrm{C}_{14} \mathrm{H}_{18} \mathrm{~N}_{4} \mathrm{O}_{3}$ & 291.1457 & 291.1437 & -6.87 & + & $\uparrow^{\star \star}$ \\
\hline 16 & 5.94 & Cortolone-3-glucuronide & $\mathrm{C}_{27} \mathrm{H}_{42} \mathrm{O}_{11}$ & 565.2625 & 565.2661 & 6.37 & + & $\downarrow^{\star \star}$ \\
\hline 17 & 2.53 & Creatine & C4H9N3O2 & 130.0617 & 130.0623 & 4.61 & - & $\downarrow^{*}$ \\
\hline 18 & 5.28 & $\begin{array}{l}\text { LysoPE (0:0/24:6 } \\
(6 Z, 9 Z, 12 Z, 15 Z, 18 Z, 21 Z))\end{array}$ & $\mathrm{C}_{29} \mathrm{H}_{48} \mathrm{NO}_{7} \mathrm{P}$ & 552.3090 & 552.3085 & -0.91 & - & $\uparrow^{*}$ \\
\hline 19 & 7.69 & Eicosapentaenoic acid & $\mathrm{C}_{20} \mathrm{H}_{30} \mathrm{O}_{2}$ & 301.2168 & 301.2188 & 6.64 & - & $\downarrow^{*}$ \\
\hline 20 & 5.93 & 12,13-Epoxy-9,15-octadecadienoic acid & $\mathrm{C}_{18} \mathrm{H}_{30} \mathrm{O}_{3}$ & 293.2117 & 293.2119 & 0.68 & - & $\uparrow^{*}$ \\
\hline 21 & 5.83 & 11,14,17-Eicosatrienoic acid & $\mathrm{C}_{20} \mathrm{H}_{34} \mathrm{O}_{2}$ & 305.2481 & 305.2500 & 6.22 & - & $\uparrow^{\star \star}$ \\
\hline
\end{tabular}

The change trend is compared between the DOX group and the NS group. $\uparrow$ and $\downarrow$ represent up- and downregulation. ${ }^{*} \mathrm{p}<0.05$. ${ }^{* *} \mathrm{p}<0.01$.

understand the metabolic or signaling pathways that these proteins may be involved in, we performed KEGG pathway (Figure 3B) annotation and found that significant changes have taken place in important pathways such as glutathione metabolism, drug metabolism-cytochrome P450, cardiac muscle contraction, fructose and mannose metabolism, glycolysis/gluconeogenesis, and complement and coagulation. However, the mechanism is still not clear and needs further clarification.

\subsection{Integrated Proteomics and Metabolomics Analysis}

To associate the proteomics data with the metabolomics data, we conducted a joint pathway analysis with differential metabolites and proteins. We found that 25 differential proteins (Table 2) were closely related to the differential metabolites. The results revealed that several metabolic pathways were significantly targeted, including fatty acid metabolism, glycolysis, amino acid metabolism, glycerol phospholipid metabolism, glutathione metabolism, and myocardial contraction (Figure 4), further relating these pathways to the occurrence of heart failure. In fatty acid metabolism, carnitine substances such as L-octanoylcarnitine and L-palmitoylcarnitine and proteins such as acetyl-CoA acyltransferase 2 and acyl-CoA dehydrogenase, $\mathrm{C}-2$ to $\mathrm{C}-3$ short chain affect the decrease of the fatty acid beta-oxidation catalytic enzyme, suggesting that the energy supply process of fatty acid $\beta$-oxidation in cardiomyocytes is affected. The upregulation of protein phosphatase and the reduction of glycolytic enzymes in glycolysis indicate that the glycolysis process of cardiomyocytes is also affected. Meanwhile, the decrease of alpha-ketoglutarate, ATP synthase, $\mathrm{H}+$ transporting, mitochondrial Fo complex, subunit F6, and succinate-CoA ligase ADP-forming beta subunit in TCA cycle indicated that the efficiency of TCA cycle was reduced. The decrease of superoxide dismutase 2, mitochondrial, cytochrome c, adenylate kinase 4, and FK506 binding protein 4 suggests the level of oxidative stress was higher. Creatine, transthyretin, and ryanodine receptor 2 decrease indicates that myocardial contraction is affected. The decrease of lysophosphatidylcholine and the increase of alpha-linolenic acid suggested that glycerol phospholipid metabolism has been affected. Elevated expression of glutathione-S-transferase represents the imbalance of glutathione metabolism in cardiomyocytes. We speculate that DOX may lead to inadequate myocardial energy supply and accumulation of cardiac reactive oxygen species through abnormal fatty acid metabolism and glucose metabolism, which together lead to calcium homeostasis affecting cardiac contractile function and lead to HF ultimately.

However, we found that most of these proteins were downstream effector proteins through KEGG analysis, so it is particularly important to find the upstream key target proteins. Then we use the protein prediction function of the STRING database to select the proteins that play an important role in proteomics results to predict the upstream proteins based on the known DOX target proteins, and we enrich the key target protein tyrosine-protein phosphatase non-receptor type 1 (PTP1B). Through the KEGG database, we can find that PTP1B can 

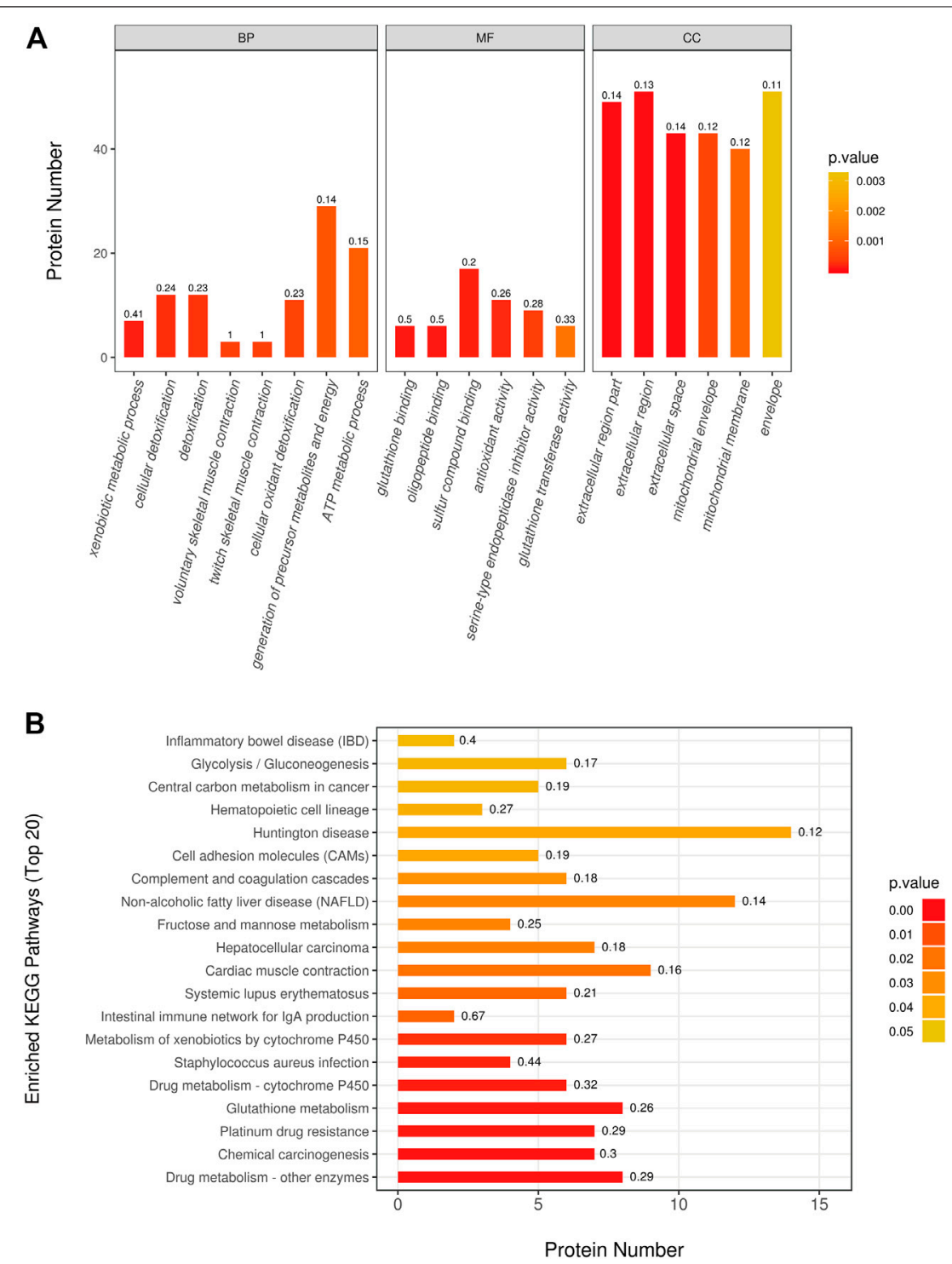

FIGURE 3 | Differentially expressed proteins pathway enrichment analysis. (A) The most significantly $(p<0.05)$ enriched GO terms (Top 20) in rat treated with doxorubicin. (B) The most significantly $(p<0.05)$ enriched KEGG pathways (Top 20) in rat treated with doxorubicin. (BP: biological process; MF: molecular function; CC: cellular component)

inhibit insulin receptor (IR) substrate and PI3K achieves GLUT4 transport by activating $\mathrm{Akt} / \mathrm{PKB}$ and $\mathrm{PKC}$ cascade and protein synthesis by mTOR and downstream components after protein kinase B activation. PP2A has an inhibitory effect on Akt and $\mathrm{PKC} \zeta$. The negative feedback signal from Akt/PKB, PKC $\zeta$, and p70S6K leads to serine phosphorylation and inactivation of IRS signal transduction, resulting in the formation of HIF- $\alpha$, and there is a certain correlation between HK2 and HIF- $\alpha$, which further affects glycolysis. When oxidative stress occurs, protein kinase $\mathrm{C}$ and phosphatidylinositol 3-kinase promote the dissociation of Nrf2 and Keap1. Nrf2 enters the nucleus and binds to antioxidant response elements to generate GSTs and SOD, thus exerting the role of antioxidant damage. With the degradation of Nrf2, the activation is terminated. AK4 and FKBP4 also affect oxidative stress, produce $\mathrm{Ttr}$ and Ryr2 to release $\mathrm{Ca} 2+$, stimulate $\mathrm{CytC}$ generation, and at the same time block the scavenging of ROS free radicals and lead to apoptosis. In Figure 5, it can be seen that PTP1B is in the upstream position of the pathway, so we speculate that it is one of the key targets upstream.

\subsection{Protein Expression in Cardiac Tissue of Two Groups}

Previous studies have shown that PTP1B may be a key upstream target for HF. To verify our hypothesis, we detected the expression of PTP1B and its downstream node proteins IRS, HIF-1 a, Nrf2, and HK-2. In results as shown in Figure 6, we found that, compared with the NS group, the expression level of PTP1B and IRS protein increased significantly, while the 
TABLE 2 | Differentially regulated proteins selected by integration analysis.

\begin{tabular}{|c|c|c|c|c|c|}
\hline No & Accession & Protein names & Gene name & LP/NS ratio & Regulated type \\
\hline 1 & ENSRNOP00000018325 & Glutathione S-transferase alpha 1 & Gsta1 & 1.31 & $\uparrow^{\star}$ \\
\hline 2 & ENSRNOP00000010579 & Microsomal glutathione S-transferase 1 & Mgst1 & 1.27 & $\uparrow^{\star \star}$ \\
\hline 3 & ENSRNOP00000024601 & Glutathione S-transferase pi 1 & Gstp1 & 1.19 & $\uparrow^{\star \star}$ \\
\hline 4 & ENSRNOP00000025939 & Glutathione S-transferase mu 2 & Gstm2 & 1.17 & $\uparrow^{\star}$ \\
\hline 5 & ENSRNOP00000039050 & Glutathione S-transferase mu 1 & Gstm1 & 1.13 & $\uparrow^{\star \star}$ \\
\hline 6 & ENSRNOP00000075572 & Glutathione S-transferase alpha 4 & Gsta4 & 1.10 & $\uparrow^{\star}$ \\
\hline 7 & ENSRNOP00000005612 & Enolase 3 & Eno3 & 0.85 & $\downarrow^{*}$ \\
\hline 8 & ENSRNOP00000018227 & Phosphoglycerate mutase 2 & Pgam2 & 0.87 & $\downarrow^{*}$ \\
\hline 9 & ENSRNOP00000008813 & Hexokinase 2 & Hk2 & 0.84 & $\downarrow^{\star *}$ \\
\hline 10 & ENSRNOP00000070507 & Phosphofructokinase, muscle & Pfkm & 0.91 & $\downarrow^{*}$ \\
\hline 11 & ENSRNOP00000022113 & Transthyretin & Ttr & 0.61 & $\downarrow^{*}$ \\
\hline 12 & ENSRNOP00000023601 & Ryanodine receptor 2 & Ryr2 & 0.87 & $\downarrow^{\star \star}$ \\
\hline 13 & ENSRNOP00000060140 & Acetyl-CoA acyltransferase 2 & Acaa2 & 0.83 & $\downarrow^{\star \star}$ \\
\hline 14 & ENSRNOP00000001556 & Acyl-CoA dehydrogenase, $\mathrm{C}-2$ to $\mathrm{C}-3$ short chain & Acads & 0.86 & $\downarrow^{\star \star}$ \\
\hline 15 & ENSRNOP00000002116 & ATP synthase, $\mathrm{H}+$ transporting, mitochondrial Fo complex, subunit F6 & Atp5pf & 0.91 & $\downarrow^{*}$ \\
\hline 16 & ENSRNOP00000060229 & Succinate-CoA ligase ADP-forming beta subunit & Sucla2 & 0.90 & $\downarrow^{*}$ \\
\hline 17 & ENSRNOP00000025794 & Superoxide dismutase 2 , mitochondrial & Sod2 & 0.84 & $\downarrow^{*}$ \\
\hline 18 & ENSRNOP00000041521 & Cytochrome c & Cyt C & 0.86 & $\downarrow^{*}$ \\
\hline 19 & ENSRNOP00000065349 & Adenylate kinase 4 & AK4 & 0.91 & $\downarrow^{\star \star}$ \\
\hline 20 & ENSRNOP00000008737 & FK506 binding protein 4 & Fkbp4 & 0.88 & $\downarrow^{*}$ \\
\hline 21 & ENSRNOP00000068826 & COX14, cytochrome c oxidase assembly factor & Cox14 & 0.85 & $\downarrow^{*}$ \\
\hline 22 & ENSRNOP00000022487 & Cytochrome c oxidase subunit 5B & Cox5b & 0.90 & $\downarrow^{*}$ \\
\hline 23 & ENSRNOP00000025525 & Cytochrome c oxidase subunit 5A & Cox5a & 0.90 & $\downarrow^{\star \star}$ \\
\hline 24 & ENSRNOP00000066702 & Cytochrome c oxidase subunit 7A1 & Cox7a1 & 0.85 & $\downarrow^{*}$ \\
\hline 25 & ENSRNOP00000023139 & Protein phosphatase 2 & PP2A & 1.10 & $\uparrow^{\star}$ \\
\hline
\end{tabular}

Pathway Diagram Key

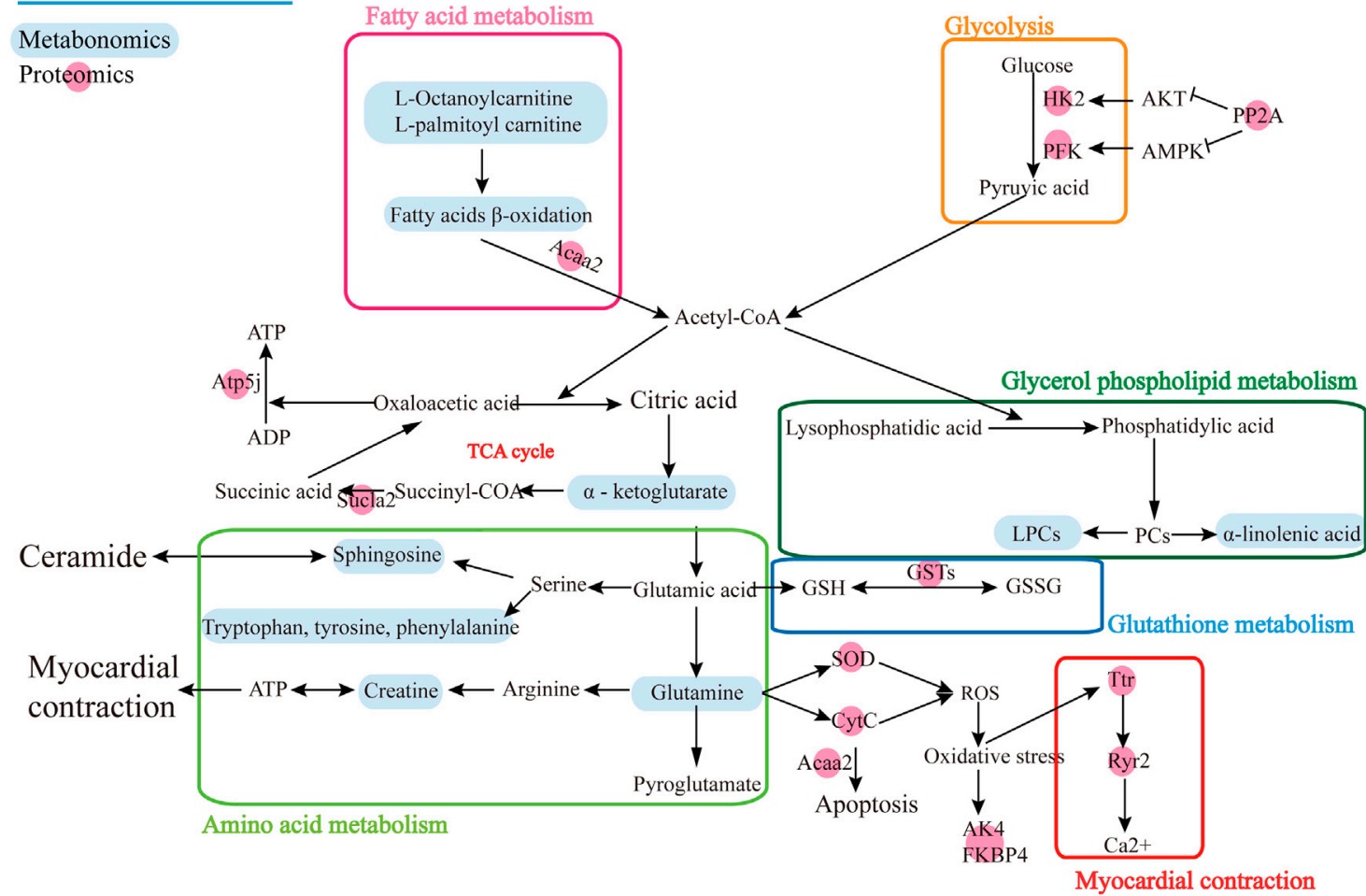

FIGURE 4 | Pathway map for combined analysis of metabolomics and proteomics. 


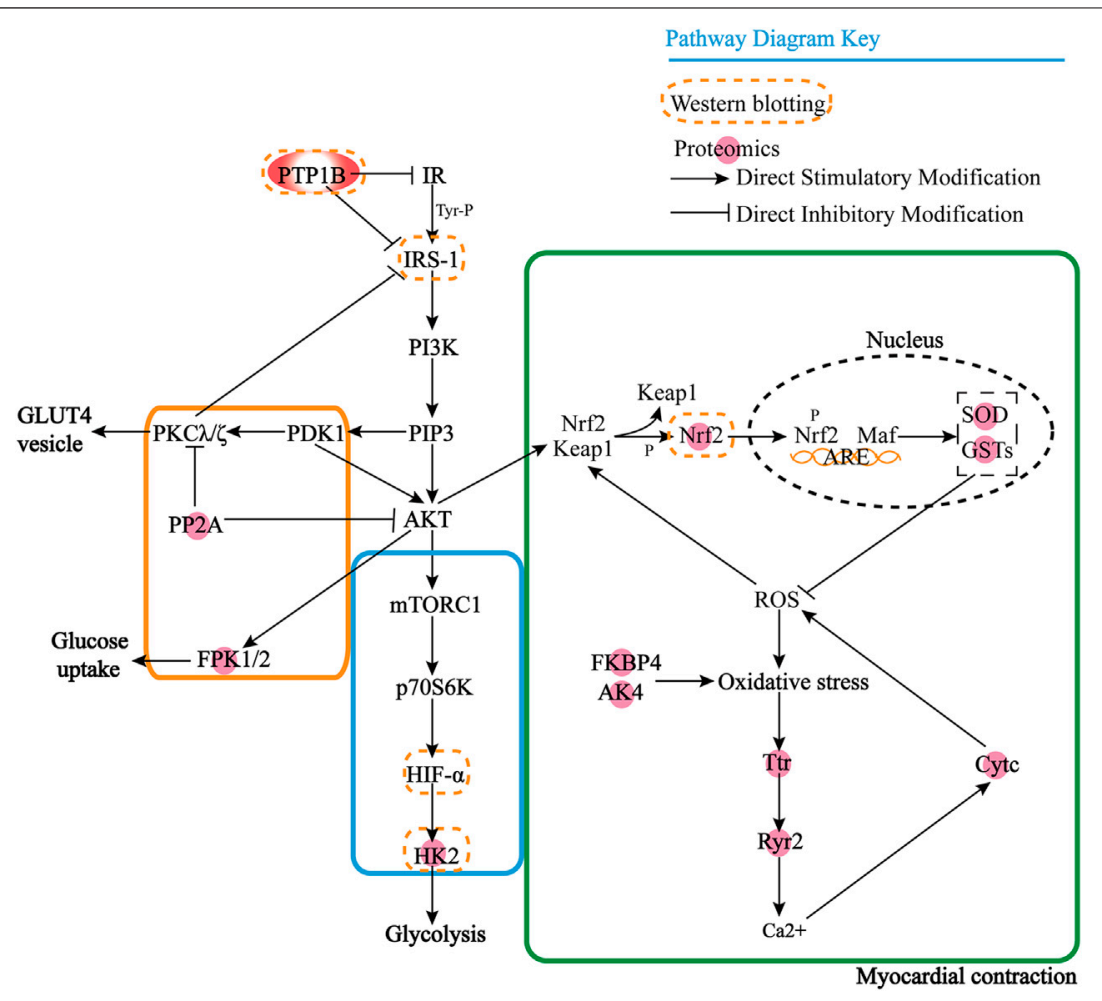

FIGURE 5 | Interaction mechanism between PTP1B and differential proteins.

A

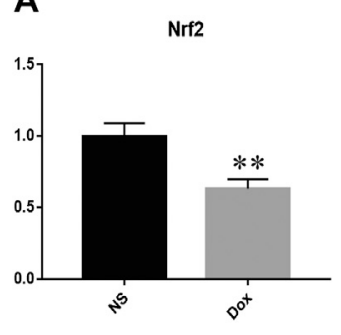

IRS-1

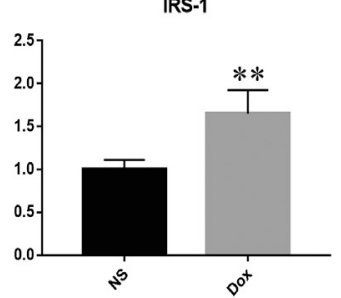

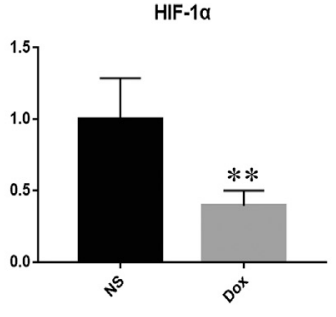

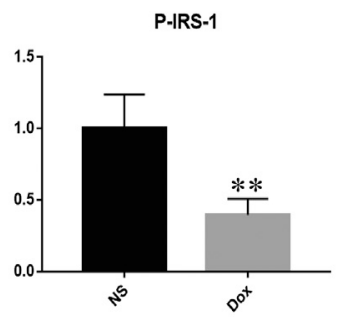

HK-2
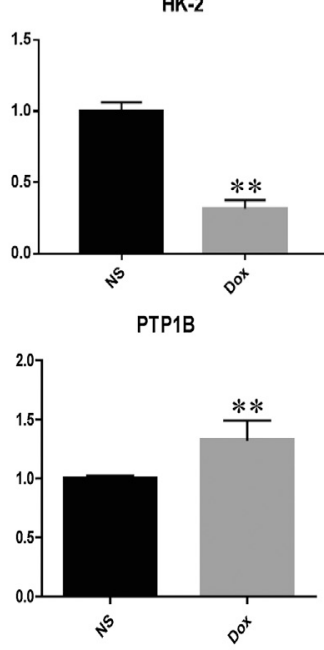

B IRS-1

P-IRS

$\mathrm{NADPH}$

Nrf2 $\rightarrow-2$
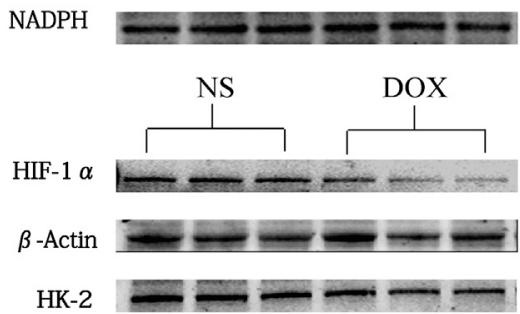

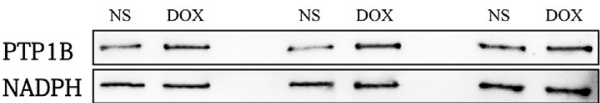

FIGURE 6 | The expression of NS group and DOX group related proteins in heart tissue $\left({ }^{*} p<0.05 ;{ }^{* *} p<0.01\right)$. (A) Expression level of PTP1B, Nrf2, HIF-1a, HK-2, IRS-1, and P-IRS in NS group and DOX group. (B) Protein level of PTP1B, Nrf2, HIF-1a, HK-2, IRS-1, and P-IRS in NS group and DOX group.

expression levels of P-IRS1, HIF-1a, Nrf2, and HK-2 protein decreased significantly in the DOX group. It is suggested that the upregulation of PTP1B protein will inhibit IRS phosphorylation, resulting in decreased P-IRS1 expression, a decrease in the expression of HIF-1a, and inhibition of downstream glycolysis; at the same time, it can also cause decreased expression of Nrf2, 
leading to increased levels of oxidative stress in the body, promoting the occurrence of HF through energy metabolism and oxidative stress.

\section{DISCUSSION}

Studies have shown that protein PTP1B, a molecular target for anti-type II diabetes, obesity, and cancer treatment, can regulate the level of protein tyrosine phosphorylation in cells (Chen et al., 2018). PTP1B is widely expressed in cardiovascular tissues, especially in the heart and endothelial cells (Thiebaut et al., 2016). Many studies have shown that inhibition of PTP1B can reduce cardiac dysfunction, systemic inflammation, and mortality (Maupoint et al., 2016; Thiebaut et al., 2016). Meanwhile, endothelial cell PTP1B deletion is associated with cardiac vascular endothelial growth factor signaling and angiogenesis and can protect against chronic afterload-induced heart failure (Gogiraju et al., 2016). Therefore, we believe that PTP1B is an interesting molecular target for the treatment of cardiovascular and metabolic diseases. However, the mechanism of PTP1B with the HF is not very clear. In our research, we found that target protein PTP1B can cause damage to the heart through energy metabolism, oxidative stress, and calcium homeostasis disorders.

\subsection{Energy Metabolism}

The heart is the most energy-consuming organ of the body and requires a large amount of ATP to provide energy. Removing mitochondrial oxidative phosphorylation, the main source of ATP is fatty acid oxidation, followed by glucose oxidation, amino acid oxidation, and so on. In heart failure, energy production is converted from fatty acid beta-oxidation to glucose oxidation, which contributes to the progressive deterioration of cardiac function in hypertrophy and heart failure (Dong et al., 2017). In this study, carnitine, Acaa2, and Acads showed a downward trend, which proved that the oxidation ability of fatty acids was affected and cardiac function was impaired (Bertero and Maack, 2018). Studies in knockdown PTP1B mice have shown that PTP1B protein has significant effects on insulin sensitivity, glucose homeostasis, and lipid metabolism (Owen et al., 2013; Zhang et al., 2016). PTP1B attenuates insulin signal transduction by removing tyrosine from activated insulin receptors and IRS- 1 triggers PI3K activation and catalyzes the lipid product phosphatidylinositol (3,4,5-) triphosphate, which in turn leads to the activation of protein kinase B to stimulate GLUT4 translocation affecting glucose absorption (Riehle and Abel, 2016; Nguyen et al., 2018; Ormazabal et al., 2018). Meanwhile, PTP1B activates mTOR through the Akt/PI3K pathway, thereby affecting HIF-1a and HK2, and converts to aerobic glycolysis metabolism (Wolf et al., 2011; Cheng et al., 2014).

Pyruvate can be oxidized by acetyl coenzyme A or as a complementary substrate to supplement the intermediate products of the TCA cycle (Turer et al., 2019). Therefore, when the rate-limiting enzyme $\mathrm{HK}-2$, which reacts with glycolytic capacity, and phosphofructokinase (Pfkp, Pfkm), which is a key regulator in glycolysis decrease, lead to a decrease in glucose utilization, pyruvate also decreases (Gibb et al., 2017; Jiao et al., 2018). Meanwhile, the level of nonessential amino acid glutamine also plays a role in regulating cellular energy homeostasis, which is converted into a-ketoglutarate, an intermediate of the citric acid cycle, by a two-step process (Tebay et al., 2015). The decrease of metabolite $\alpha$-ketoglutaric acid, differential protein atp $5 \mathrm{j}$, and sucla 2 indicated the decrease of TCA cycle efficiency. This reflects the reduced TCA cycle efficiency of cardiomyocytes and the inability to provide sufficient ATP, indicating that fatty acid oxidation and glycolysis can affect the downstream TCA cycle, resulting in a decrease in ATP.

In the study, we found that the LPCs decreased significantly, which has been demonstrated to be accompanied by a decrease in ejection fraction when cardiovascular disease occurrs, and a decrease in $\mathrm{EF}$ is associated with disorders in phospholipid metabolism (Marcinkiewicz-Siemion et al., 2018). Christin Stegemann et al. (Stegemann et al., 2014) analyzed the relationship between molecular lipids and cardiovascular risk and found that LPCs, cholesterol esters, phosphatidylcholine, phosphatidylethanolamine, sphingomyelin, and triacylglycerol were associated with cardiovascular disease. Combined with the above factors, PTP1B protein affected the phosphorylation of insulin receptor substrate protein IRS, caused disorders of glycolysis and lipid metabolism, and was accompanied by the imbalance of glutamine regulatory capacity homeostasis, which together led to the impact of TCA cycle, resulting in the reduction of cardiac energy supply.

\subsection{Oxidative Stress}

Oxidative stress is an important feature of the onset and development of many diseases (including cardiovascular diseases), and one of the common features that play an important role in the pathophysiology of heart failure is chronic oxidative stress (Belch et al., 1991; Hill and Singal, 1997; Zima and Mazurek, 2016). PTP1B increases glutathione S-transferase (GSTs) protein levels by stimulating insulin receptor substrates, activating downstream PI3K, Akt/protein kinase B, ribosomal p70S6 kinase, and PKC (Kim et al., 2006). Gamma 1-glutamyl-1-cysteine glycine (GSH), a tripeptide that can prevent oxidative stress and can be oxidized to form glutathione two sulfur (GSSG), when the ratio between oxidized and reduced GSH increases and glutathione is mixed with disulfide, will play a pathogenic factor in cardiovascular diseases (Lu, 2013; Zima and Mazurek, 2016; Chevallier et al., 2020) The increased expression of glutathione S-transferase represents the imbalance of glutathione metabolism in cardiomyocytes, depletion of GSH increases and the ratio of GSH/GSSG decreases, which enhanced oxidative stress from ROS in cardiomyocytes (Cramer et al., 2017; Ma et al., 2018). Among them, Gstp1 can sensitize cells to free radical-mediated damage by reducing the ability of reactive electrophiles to bind to glutathione, and its main active site is Val105, which is mainly associated with cardiovascular diseases (Doney et al., 2005). The GSTM family is associated with breast cancer, and there is a certain association between breast cancer and oxidative stress ( $\mathrm{Li}$ 
et al., 2018). Anthracyclines are also metabolized by GSTMsmediated reactions, which can accelerate the metabolic inactivation of therapeutic drugs (Tan et al., 2008). GSTA4 is a 2-phase detoxifying enzyme whose expression increases in response to oxidative stress (Shearn et al., 2016). In this study, Gsta1/4, Gstm1/2, and Gstp1GSTs all showed a significant upward trend, demonstrating that doxorubicin-induced heart failure has a strong correlation with oxidative stress.

On the other hand, Sharma Sudha et al. demonstrated that the absence of SOD2 leads to an increase in ROS (Sharma et al., 2020). Nrf2 is widely recognized as a transcription factor activated by oxidative stress, and cells lacking Nrf2 can lead to mitochondrial dysfunction, resulting in increased ROS and impaired antioxidant capacity and further aggravated heart failure (Tebay et al., 2015). Nrf2 plays a crucial role in activating the Sirt3/SOD2 signaling pathway, and studies have demonstrated significant inhibition of silenced Nrf2 cells, SOD2 activity, and GSH/GSSG ratio, as well as increased ROS and MDA levels in induced oxidative stress cell models (Zhou et al., 2019). Abnormal cell membrane potential leads cytochrome C (CytC) to the cytoplasm and enhances apoptosis (Sun Jang et al., 2009; Zhang et al., 2019). And the increase of ROS level will inhibit superoxide dismutase (SOD), catalase (Marwick), GSH-PX activity, and GSH content (Liu et al., 2020). Overexpression of AK4 stabilizes HIF-1a protein by increasing intracellular ROS levels (Jan et al., 2019). In this study, AK4 and HIF-1a showed a downward trend, while Nrf2, SOD, and CytC showed a significant downward trend, indicating that oxidative stress played a crucial role in heart failure. Therefore, GSH, Nrf2, SOD2, and CytC are related to the occurrence of heart failure during oxidative stress.

\subsection{Calcium Homeostasis}

Calcium homeostasis is a central point in maintaining normal cardiac contractility in heart failure. Dysfunction of systolic $\mathrm{Ca}$ transport caused by dysfunction of the type 2 ryanodine receptor (RyR2) in the sarcoplasmic reticulum is associated with a variety of heart diseases, including catecholaminergic polymorphic ventricular tachycardia, atrial fibrillation, and HF (Connell et al., 2020). In cardiomyocytes, Ca2+ enters through L-type $\mathrm{Ca} 2+$ channels, which mediate the opening of RyR2 channels, allowing $\mathrm{Ca} 2+$ to contract from sarcoplasmic reticulum to cytoplasm, thus releasing energy from ATP hydrolysis by binding to enzymes on mitochondria (Kaplan et al., 2003; Gonano and Vila Petroff, 2020). Suetomi et al. studies have shown that when RyR2 deficiency or expression decreases, $\mathrm{Ca} 2+$ spontaneously leaks from the sarcoplasmic reticulum (Suetomi et al., 2011), which influences the calcium homeostasis of cardiomyocytes, reduces the production of ATP from mitochondria, and leads to heart failure due to cardiac systolic dysfunction. This study demonstrates that oxidative stress leads to the expression of Ttr protein (Zhang P. et al., 2018), which subsequently affects the low expression of RyR2, leading to $\mathrm{Ca} 2+$ homeostasis disorders affecting myocardial contraction. FK506-binding protein 52 (FKBP52, also known as FKBP4), whose downregulation is associated with cardiomyocyte hypertrophy, regulates $\mathrm{Ca} 2+$ signaling in a manner dependent on overexpression of peptidyl-prolyl isomerase (PPIase) (Bandleon et al., 2019).

Also, ATP derived from the conversion of phosphokinase to creatine is an important chemical source of myocardial contraction, and the downregulation of creatine also indicates that cardiac systolic and diastolic function is affected during heart failure (Haris et al., 2014).

\section{CONCLUSION}

The combined analysis results showed that the occurrence of heart failure was mainly related to the metabolic disorders of fatty acid metabolism, glycolysis, TCA cycle, glycerophospholipid metabolism, glutathione metabolism, and amino acid metabolism. These metabolic pathways are closely related to energy metabolism, oxidative stress, and myocardial contraction. In this study, we focused on the discussion that PTP1B inhibits the expression of hypoxiainducible factor-1 alpha (HIF-1a) by inhibiting the phosphorylation of IRS, leading to the disorder of fatty acid metabolism and glycolysis; on the other hand, the increased expression of GSTs, which leads to the decrease of GSH/GSSG ratio and the decrease of Nrf2, SOD, Cytc, and AK4 together lead to oxidative stress. Decreased expression of Ryr2 results in abnormal cardiac contraction with abnormal $\mathrm{Ca} 2+$ homeostasis and decreased ATP. Heart failure is usually defined as a condition of chronic oxidative stress due to energy shortage, so this study suggests that the occurrence of heart failure may be related to a harmful cycle of calcium homeostasis disorders resulting from increased energy demand and increased workload in HF (Seddon et al., 2007; Zima and Mazurek, 2016). It provides a new strategy for the effective treatment of heart failure, the development of new drugs, and the study of pharmacodynamic material basis, but in this study, only the important target protein $\mathrm{PTP} 1 \mathrm{~B}$ and the subsequent pathway of heart failure have been preliminarily validated; therefore, further functional validation of the target protein is needed from the cellular and animal levels.

Compared with other omics, data generated by proteomics and metabolomics are more directly related to the pathological symptoms and clinical parameters observed by patients (Rinschen and Saez-Rodriguez, 2020). Proteomics remains the most commonly used tool for discovering new biomarkers (Montaner et al., 2020). At present, the combination of multiomics has become a hot topic in the study of disease mechanism. From a clinical point of view, blood has more easily acquired advantages and plasma/serum metabolomics integrated systemic metabolism has better diagnostic and prognostic value than conventional biomarkers (Cheng et al., 2015; Hunter et al., 2016; McGarrah et al., 2018). In this study, we found the changes of metabolites and proteins during heart failure in rats by metabolomics and proteomics, explained the mechanism of heart failure at the phenotypic and genotypic levels, and provided a reference for the study of DOX-induced heart failure. However, in order to provide more information related to the heart's own metabolism, we need further studies of tissue metabolomics to reflect molecular processes closer to the disease state. 


\section{DATA AVAILABILITY STATEMENT}

The original contributions presented in the study are included in the article/Supplementary Material, further inquiries can be directed to the corresponding authors.

\section{ETHICS STATEMENT}

The animal study was reviewed and approved by the Institutional Animal Care and Use Committee of Tianjin University of Traditional Chinese Medicine (IACUC), and was conducted in accordance to the guidelines of the National Institutes of Health Animal Care and Use Committee.

\section{AUTHOR CONTRIBUTIONS}

YW and YL planned and supervised the experiments. SF, WH, YY, YW, and LS analyzed and interpretated the data. YY and YW wrote the article. All authors agreed to the publication. The authors read and approved the final manuscript.

\section{REFERENCES}

Bandleon, S., Strunz, P. P., Pickel, S., Tiapko, O., Cellini, A., Miranda-Laferte, E., et al. (2019). FKBP52 regulates TRPC3-dependent $\mathrm{Ca}^{2+}$ signals and the hypertrophic growth of cardiomyocyte cultures. J. Cell Sci. 132, 231506. doi: $10.1242 /$ jcs. 231506

Belch, J. J., Bridges, A. B., Scott, N., and Chopra, M. (1991). Oxygen free radicals and congestive heart failure. Heart 65, 245-248. doi:10.1136/hrt.65.5.245

Bertero, E. and Maack, C. (2018). Metabolic remodelling in heart failure. Nat. Rev. Cardiol. 15, 457-470. doi:10.1038/s41569-018-0044-6

Chen, X., Gan, Q., Feng, C., Liu, X., and Zhang, Q. (2018). Virtual screening of novel and selective inhibitors of protein tyrosine phosphatase $1 \mathrm{~B}$ over T-cell protein tyrosine phosphatase using a bidentate inhibition strategy. J. Chem. Inf. Model. 58, 837-847. doi:10.1021/acs.jcim.8b00040

Cheng, M.-L., Wang, C.-H., Shiao, M.-S., Liu, M.-H., Huang, Y.-Y., Huang, C.-Y., et al. (2015). Metabolic disturbances identified in plasma are associated with outcomes in patients with heart failure. J. Am. Coll. Cardiol. 65, 1509-1520. doi:10.1016/j.jacc.2015.02.018

Cheng, S.-C., Quintin, J., Cramer, R. A., Shepardson, K. M., Saeed, S., Kumar, V., et al. (2014). mTOR- and HIF-1 -mediated aerobic glycolysis as metabolic basis for trained immunity. Science 345, 1250684. doi:10.1126/science.1250684

Chevallier, V., Andersen, M. R., and Malphettes, L. (2020). Oxidative stressalleviating strategies to improve recombinant protein production in $\mathrm{CHO}$ cells. Biotechnol. Bioeng. 117, 1172-1186. doi:10.1002/bit.27247

Connell, P., Word, T. A., and Wehrens, X. H. T. (2020). Targeting pathological leak of ryanodine receptors: preclinical progress and the potential impact on treatments for cardiac arrhythmias and heart failure. Expert Opin. Ther. Targets 24, 25-36. doi:10.1080/14728222.2020.1708326

Cramer, S. L., Saha, A., Liu, J., Tadi, S., Tiziani, S., Yan, W., et al. (2017). Systemic depletion of L-cyst(e)ine with cyst(e)inase increases reactive oxygen species and suppresses tumor growth. Nat. Med. 23, 120-127. doi: $10.1038 / \mathrm{nm} .4232$

Doney, A. S. F., Lee, S., Leese, G. P., Morris, A. D., and Palmer, C. N. A. (2005). Increased cardiovascular morbidity and mortality in type 2 diabetes is associated with the glutathione S transferase theta-null genotype. Circulation 111, 2927-2934. doi:10.1161/circulationaha.104.509224

\section{FUNDING}

This work was supported by the Tianjin Development Program for Innovation and Entrepreneurship and National Natural Science Foundation of China (No. 81903938).

\section{ACKNOWLEDGMENTS}

This paper has been released as a pre-print at Research Square, Exploration the mechanism of doxorubicin-induced heart failure in rats by integration of proteomics and metabolomics data (https://doi. org/10.21203/rs.3.rs-58887/v1) (Yuan et al., 2020). The authors are grateful to the members of the Shanghai Applied Protein Technology Co., Ltd. (Shanghai, China), for tandem mass spectrometry (TMT) quantitative proteomics analysis and data interpretation.

\section{SUPPLEMENTARY MATERIAL}

The Supplementary Material for this article can be found online at: https://www.frontiersin.org/articles/10.3389/fphar.2020.600561/ full\#supplementary-material

Dong, Z., Zhao, P., Xu, M., Zhang, C., Guo, W., Chen, H., et al. (2017). Astragaloside IV alleviates heart failure via activating PPARa to switch glycolysis to fatty acid $\beta$-oxidation. Sci. Rep. 7, 2691. doi:10.1038/s41598017-02360-5

Eisenberg, E., Di Palo, K. E., and Piña, I. L., (2018). Sex differences in heart failure. Clin. Cardiol. 41, 211-216. doi:10.1002/clc.22917

Feijen, E. A. M., Leisenring, W. M., Stratton, K. L., Ness, K. K., van der Pal, H. J. H., van Dalen, E. C., et al. (2019). Derivation of anthracycline and anthraquinone equivalence ratios to doxorubicin for late-onset cardiotoxicity. JAMA Oncol. 5, 864-871. doi:10.1001/jamaoncol.2018.6634

Fu, H. Y., Sanada, S., Matsuzaki, T., Liao, Y., Okuda, K., Yamato, M., et al. (2016). Chemical endoplasmic reticulum chaperone alleviates doxorubicin-induced cardiac dysfunction. Circ. Res. 118, 798-809. doi:10.1161/circresaha.115.307604

Galán-Arriola, C., Lobo, M., Vílchez-Tschischke, P. J., López, G. J., de MolinaIracheta, A., Pérez-Martínez, C., et al. (2019). Serial magnetic resonance imaging to identify early stages of anthracycline-induced cardiotoxicity. J. Am. Coll. Cardiol. 73, 779-791. doi:10.1016/j.jacc.2018.11.046

Gibb, A. A., Lorkiewicz, P. K., Zheng, Y.-T., Zhang, X., Bhatnagar, A., Jones, S. P., et al. (2017). Integration of flux measurements to resolve changes in anabolic and catabolic metabolism in cardiac myocytes. Biochem. J. 474, 2785-2801. doi:10.1042/bcj20170474

Gogiraju, R., Schroeter, M. R., Bochenek, M. L., Hubert, A., Münzel, T., Hasenfuss, G., et al. (2016). Endothelial deletion of protein tyrosine phosphatase-1B protects against pressure overload-induced heart failure in mice. Cardiovasc. Res. 111, 204-216. doi:10.1093/cvr/cvw101

Gonano, L. A. and Vila Petroff, M. (2020). Direct modulation of RyR2 leading to a TRICky Ca 2+ balance. Circ. Res. 126, 436-438. doi:10.1161/circresaha.120. 316532

Haris, M., Singh, A., Cai, K., Kogan, F., McGarvey, J., DeBrosse, C., et al. (2014). A technique for in vivo mapping of myocardial creatine kinase metabolism. Nat. Med. 20, 209-214. doi:10.1038/nm.3436

Hill, M. F. and Singal, P. K. (1997). Right and left myocardial antioxidant responses during heart failure subsequent to myocardial infarction. Circulation 96, 2414-2420. doi:10.1161/01.cir.96.7.2414

Hoffman, J., Lyu, Y., Pletcher, S. D., and Promislow, D. E. L. (2017). Proteomics and metabolomics in ageing research: from biomarkers to systems biology. Essays Biochem. 61, 379-388. doi:10.1042/EBC20160083 
Hunter, W. G., Kelly, J. P., McGarrah, R. W., Kraus, W. E., and Shah, S. H. (2016). Metabolic dysfunction in heart failure: diagnostic, prognostic, and pathophysiologic insights from metabolomic profiling. Curr. Heart Fail. Rep. 13, 119-131. doi:10.1007/s11897-016-0289-5

Jan, Y., Lai, T., Yang, C., Lin, Y., Huang, M., and Hsiao, M. (2019). Adenylate kinase 4 modulates oxidative stress and stabilizes HIF-1a to drive lung adenocarcinoma metastasis. J. Hematol. Oncol. 12, 12. doi:10.1186/s13045019-0698-5

Jiao, L., Zhang, H.-L., Li, D.-D., Yang, K.-L., Tang, J., Li, X., et al. (2018). Regulation of glycolytic metabolism by autophagy in liver cancer involves selective autophagic degradation of HK2 (hexokinase 2). Autophagy 14, 671-684. doi:10.1080/15548627.2017.1381804

Kaplan, P., Babusikova, E., Lehotsky, J., and Dobrota, D. (2003). Free radicalinduced protein modification and inhibition of Ca2+-ATPase of cardiac sarcoplasmic reticulum. Mol. Cell Biochem. 248, 41-47. doi:10.1023/a: 1024145212616

Kim, S. K., Abdelmegeed, M. A., and Novak, R. F. (2006). Identification of the insulin signaling cascade in the regulation of alpha-class glutathione S-transferase expression in primary cultured rat hepatocytes. J. Pharmacol. Exp. Therapeut. 316, 1255-1261. doi:10.1124/jpet.105.096065

Li, S., Lang, G. T., Zhang, Y. Z., Yu, K. D., Shao, Z. M., and Zhang, Q. (2018). Interaction between glutathione S-transferase M1-null/present polymorphism and adjuvant chemotherapy influences the survival of breast cancer. Cancer Med. 7, 4202-4207. doi:10.1002/cam4.1567

Liu, Q., Wang, W., Zhang, Y., Cui, Y., Xu, S., and Li, S. (2020). Bisphenol A regulates cytochrome $\mathrm{P} 450 \mathrm{~B} 1$ through miR-27b-3p and induces carp lymphocyte oxidative stress leading to apoptosis. Fish Shellish Immunol. 102, 489-498. doi:10.1016/j.fsi.2020.05.009

Lu, S. C. (2013). Glutathione synthesis. Biochim. Biophys. Acta Gen. Subj. 1830, 3143-3153. doi:10.1016/j.bbagen.2012.09.008

Ma, R., Ji, T., Zhang, H., Dong, W., Chen, X., Xu, P., et al. (2018). A Pck1-directed glycogen metabolic program regulates formation and maintenance of memory CD8+ T cells. Nat. Cell Biol. 20, 21-27. doi:10.1038/s41556-017-0002-2

Marcinkiewicz-Siemion, M., Ciborowski, M., Ptaszynska-Kopczynska, K., Szpakowicz, A., Lisowska, A., Jasiewicz, M., et al. (2018). LC-MS-based serum fingerprinting reveals significant dysregulation of phospholipids in chronic heart failure. J. Pharmaceut. Biomed. Anal. 154, 354-363. doi:10. 1016/j.jpba.2018.03.027

Marwick, T. H. (2015). The role of echocardiography in heart failure. J. Nucl. Med. 56, 31-38. doi:10.2967/jnumed.114.150433

Mato, J. M., Martínez-Chantar, M. L., and Lu, S. C. (2014). Systems biology for hepatologists. Hepatology 60, 736-743. doi:10.1002/hep.27023

Maupoint, J., Besnier, M., Gomez, E., Bouhzam, N., Henry, J.-P., Boyer, O., et al. (2016). Selective vascular endothelial protection reduces cardiac dysfunction in chronic heart failure. Circ. Heart Fail. 9, e002895. doi:10.1161/circheartfailure. 115.002895

Maurer, M. and Packer, M. (2020). How should physicians assess myocardial contraction?: redefining heart failure with a preserved ejection fraction. JACC Cardiovasc. Imaging 13, 873-878. doi:10.1016/j.jcmg.2019.12.021

McGarrah, R. W., Crown, S. B., Zhang, G.-F., Shah, S. H., and Newgard, C. B. (2018). Cardiovascular metabolomics. Circ. Res. 122, 1238-1258. doi:10.1161/ circresaha.117.311002

Montaner, J., Ramiro, L., Simats, A., Tiedt, S., Makris, K., Jickling, G. C., et al. (2020). Multilevel omics for the discovery of biomarkers and therapeutic targets for stroke. Nat. Rev. Neurol. 16, 247-264. doi:10.1038/s41582-020-0350-6

Nguyen, T., Schwarzer, M., Schrepper, A., Amorim, P. A., Blum, D., Hain, C., et al. (2018). Increased protein tyrosine phosphatase 1B (PTP1B) activity and cardiac insulin resistance precede mitochondrial and contractile dysfunction in pressure-overloaded hearts. J. Am. Heart Assoc. 7, e008865. doi:10.1161/ JAHA.118.008865

Ormazabal, P., Scazzocchio, B., Varì, R., Santangelo, C., D’Archivio, M., Silecchia, G., et al. (2018). Effect of protocatechuic acid on insulin responsiveness and inflammation in visceral adipose tissue from obese individuals: possible role for PTP1B. Int. J. Obes. 42, 2012-2021. doi:10.1038/s41366-018-0075-4

Owen, C., Lees, E. K., Grant, L., Zimmer, D. J., Mody, N., Bence, K. K., et al. (2013). Inducible liver-specific knockdown of protein tyrosine phosphatase $1 \mathrm{~B}$ improves glucose and lipid homeostasis in adult mice. Diabetologia 56, 2286-2296. doi:10.1007/s00125-013-2992-z
Riehle, C. and Abel, E. D. (2016). Insulin signaling and heart failure. Circ. Res. 118, 1151-1169. doi:10.1161/circresaha.116.306206

Rinschen, M. and Saez-Rodriguez, J. (2020). The tissue proteome in the multiomic landscape of kidney disease. Nat. Rev. Nephrol. doi:10.1038/s41581020-00348-5

Rochette, L., Guenancia, C., Gudjoncik, A., Hachet, O., Zeller, M., Cottin, Y., et al. (2015). Anthracyclines/trastuzumab: new aspects of cardiotoxicity and molecular mechanisms. Trends Pharmacol. Sci. 36, 326-348. doi:10.1016/j. tips.2015.03.005

Russo, M., Guida, F., Paparo, L., Trinchese, G., Aitoro, R., Avagliano, C., et al. (2019). The novel butyrate derivative phenylalanine-butyramide protects from doxorubicin-induced cardiotoxicity. Eur. J. Heart Fail. 21, 519-528. doi:10. 1002/ejhf.1439

Seddon, M., Looi, Y. H., and Shah, A. M. (2007). Oxidative stress and redox signalling in cardiac hypertrophy and heart failure. Heart 93, 903-907. doi:10. 1136/hrt.2005.068270

Sharma, S., Bhattarai, S., Ara, H., Sun, G., St Clair, D. K., Bhuiyan, M. S., et al. (2020). SOD2 deficiency in cardiomyocytes defines defective mitochondrial bioenergetics as a cause of lethal dilated cardiomyopathy. Redox Biol. 37, 101740. doi:10.1016/j.redox.2020.101740

Shearn, C. T., Fritz, K. S., Shearn, A. H., Saba, L. M., Mercer, K. E., Engi, B., et al. (2016). Deletion of GSTA4-4 results in increased mitochondrial posttranslational modification of proteins by reactive aldehydes following chronic ethanol consumption in mice. Redox Biol. 7, 68-77. doi:10.1016/j. redox.2015.11.013

Stegemann, C., Pechlaner, R., Willeit, P., Langley, S. R., Mangino, M., Mayr, U., et al. (2014). Lipidomics profiling and risk of cardiovascular disease in the prospective population-based Bruneck study. Circulation 129, 1821-1831. doi:10.1161/circulationaha.113.002500

Suetomi, T., Yano, M., Uchinoumi, H., Fukuda, M., Hino, A., Ono, M., et al. (2011). Mutation-linked defective interdomain interactions within ryanodine receptor cause aberrant $\mathrm{Ca} 2+$ release leading to catecholaminergic polymorphic ventricular tachycardia. Circulation 124, 682-694. doi:10.1161/ circulationaha.111.023259

Sun Jang, J., Piao, S., Cha, Y.-N., and Kim, C. (2009). Taurine chloramine activates Nrf2, increases HO-1 expression and protects cells from death caused by hydrogen peroxide. J. Clin. Biochem. Nutr. 45, 37-43. doi:10.3164/jcbn.08-262

Tan, S., Lee, S.-C., Goh, B.-C., and Wong, J. (2008). Pharmacogenetics in breast cancer therapy. Clin. Cancer Res. 14, 8027-8041. doi:10.1158/1078-0432.CCR08-0993

Tebay, L. E., Robertson, H., Durant, S. T., Vitale, S. R., Penning, T. M., DinkovaKostova, A. T., et al. (2015). Mechanisms of activation of the transcription factor Nrf2 by redox stressors, nutrient cues, and energy status and the pathways through which it attenuates degenerative disease. Free Radic. Biol. Med. 88, 108-146. doi:10.1016/j.freeradbiomed.2015.06.021

Thiebaut, P.-A., Besnier, M., Gomez, E., and Richard, V. (2016). Role of protein tyrosine phosphatase 1B in cardiovascular diseases. J. Mol. Cell. Cardiol. 101, 50-57. doi:10.1016/j.yjmcc.2016.09.002

Turer, A., Altamirano, F., Schiattarella, G. G., May, H., Gillette, T. G., Malloy, C. R., et al. (2019). Remodeling of substrate consumption in the murine sTAC model of heart failure. J. Mol. Cell. Cardiol. 134, 144-153. doi:10.1016/j.yjmcc.2019.07. 007

Wolf, A., Agnihotri, S., Micallef, J., Mukherjee, J., Sabha, N., Cairns, R., et al. (2011). Hexokinase 2 is a key mediator of aerobic glycolysis and promotes tumor growth in human glioblastoma multiforme. J. Exp. Med. 208, 313-326. doi:10. 1084/jem.20101470

Yuan, Y., Fan, S., Shu, L., Huang, W., Xie, L., Bi, C., et al. (2020). Exploration the mechanism of doxorubicin-induced heart failure in rats by integration of proteomics and metabolomics data. Res. Square, 1-24. doi:10.21203/rs.3. rs-58887/v1

Zhang, H., Xu, A., Sun, X., Yang, Y., Zhang, L., Bai, H., et al. (2020). Selfmaintenance of cardiac resident reparative macrophages attenuates doxorubicin-induced cardiomyopathy through the SR-A1-C-MYC axis. Circ. Res. 127, 610-627. doi:10.1161/circresaha.119.316428

Zhang, K. W., Finkelman, B. S., Gulati, G., Narayan, H. K., Upshaw, J., Narayan, V., et al. (2018). Abnormalities in 3-dimensional left ventricular mechanics with anthracycline chemotherapy are associated with systolic and diastolic dysfunction. J. Am. Coll. Cardiol. 11, 1059-1068. doi:10.1016/j.jcmg.2018.01.015 
Zhang, P., Guan, X., Yang, M., Zeng, L., and Liu, C. (2018). Roles and potential mechanisms of selenium in countering thyrotoxicity of DEHP. Sci. Total Environ. 619-620, 732-739. doi:10.1016/j.scitotenv.2017.11.169

Zhang, X., Tian, J., Li, J., Huang, L., Wu, S., Liang, W., et al. (2016). A novel protein tyrosine phosphatase $1 \mathrm{~B}$ inhibitor with therapeutic potential for insulin resistance. Br. J. Pharmacol. 173, 1939-1949. doi:10.1111/bph. 13483

Zhang, Y., Wang, X., Chen, C., An, J., Shang, Y., Li, H., et al. (2019). Regulation of TBBPA-induced oxidative stress on mitochondrial apoptosis in L02 cells through the Nrf2 signaling pathway. Chemosphere 226, 463-471. doi:10. 1016/j.chemosphere.2019.03.167

Zhou, Q., Wang, X., Shao, X., Wang, H., Liu, X., Ke, X., et al. (2019). Tertbutylhydroquinone treatment alleviates contrast-induced nephropathy in rats by activating the Nrf2/Sirt3/SOD2 signaling pathway. Oxid. Med. Cell Longev. 2019, 4657651. doi:10.1155/2019/4657651
Zima, A. V. and Mazurek, S. R. (2016). Functional impact of ryanodine receptor oxidation on intracellular calcium regulation in the heart. Rev. Physiol. Biochem. Pharmacol. 171, 39-62. doi:10.1007/112_2016_2

Conflict of Interest: The authors declare that the research was conducted in the absence of any commercial or financial relationships that could be construed as a potential conflict of interest.

Copyright (c) 2020 Yuan, Fan, Shu, Huang, Xie, Bi, Yu, Wang and Li. This is an open-access article distributed under the terms of the Creative Commons Attribution License (CC BY). The use, distribution or reproduction in other forums is permitted, provided the original author(s) and the copyright owner(s) are credited and that the original publication in this journal is cited, in accordance with accepted academic practice. No use, distribution or reproduction is permitted which does not comply with these terms. 